\title{
Multiple Attribute Decision Making Based on Generalized Aggregation Operators under Dual Hesitant Fuzzy Environment
}

\author{
Chunyong Wang, ${ }^{1}$ Qingguo Li, ${ }^{1}$ and Xiaoqiang Zhou ${ }^{1,2}$ \\ ${ }^{1}$ College of Mathematics and Econometrics, Hunan University, Changsha, Hunan 410082, China \\ ${ }^{2}$ College of Mathematics, Hunan Institute of Science and Technology, Yueyang, Hunan 414006, China \\ Correspondence should be addressed to Qingguo Li; liqingguoli@aliyun.com
}

Received 4 January 2014; Accepted 27 March 2014; Published 4 May 2014

Academic Editor: Francis T. K. Au

Copyright (c) 2014 Chunyong Wang et al. This is an open access article distributed under the Creative Commons Attribution License, which permits unrestricted use, distribution, and reproduction in any medium, provided the original work is properly cited.

\begin{abstract}
We investigate the multiple attribute decision making (MADM) problems with dual hesitant fuzzy information. We first introduce some basic concepts and operations on dual hesitant fuzzy sets. Then, we develop some generalized dual hesitant fuzzy aggregation operators which encompass some existing operators as their particular cases and discuss their basic properties. Next, we apply the generalized dual hesitant fuzzy Choquet ordered aggregation (GDHFCOA) operator to deal with multiple attribute decision making problems under dual hesitant fuzzy environment. Finally, an illustrative example is given to show the developed method and demonstrate its practicality and effectiveness.
\end{abstract}

\section{Introduction}

Fuzzy set (FS), which is proposed by Zadeh [1], is a powerful tool to deal with vagueness or nonstatistical imprecision. Since its appearance, it has received much attention and wide applications [2-6]. Many celebrated extensions have been developed including type-2 fuzzy sets (T2FSs) [7], fuzzy multisets (FMSs) $[8,9]$, intuitionistic fuzzy sets (IFSs) [10], interval-valued intuitionistic fuzzy sets (IVIFSs) [11, 12], and hesitant fuzzy sets (HFSs) [13-17].

Recently, Zhu et al. [18] introduced a dual hesitant fuzzy set (DHFS) which is another new extension of FSs. It is a comprehensive set containing FSs, IFSs, FMSs, and HFSs as special cases under certain conditions. By several possible values for the membership and nonmembership degrees, respectively, DHFSs can take much more information given by decision makers into account in multiple attribute decision making. In their work, some basic operations and properties for DHFSs were investigated. Then Wang et al. [19] investigated the multiple attribute decision making (MADM) problem based on the aggregation operators with dual hesitant fuzzy information. They also developed some aggregation operators for aggregating dual hesitant fuzzy information including dual hesitant fuzzy weighted average (DHFWA) operator, dual hesitant fuzzy weighted geometric (DHFWG) operator, dual hesitant fuzzy ordered weighted average (DHFOWA) operator, dual hesitant fuzzy ordered weighted geometric (DHFOWG) operator, dual hesitant fuzzy hybrid average (DHFHA) operator, and dual hesitant fuzzy hybrid geometric (DHFHG) operator.

However, the existing dual hesitant fuzzy aggregation operators above only consider situations where all the attributes in the dual hesitant fuzzy set are independent. Nevertheless, attributes in DHFSs are usually correlative in real life. Incidentally, the Choquet integral [20] can characterize the correlations of the decision data. Motivated by this idea, we propose a dual hesitant fuzzy Choquet ordered aggregation (DHFCOA) operator, whose prominent characteristic is that it can consider both the importance of the attributes and the correlations of the attributes. It is worth mentioning that DHFCOA can be regarded as an extension of DHFWA and DHFOWA. Then, we also generate DHFCOA operator to GDHFCOA.

To do so, the remainder of this paper is organized as follows. In the next section, we introduce some basic concepts related to dual hesitant fuzzy sets, as well as the 
existing dual hesitant fuzzy aggregation operators. Some generalized aggregation operators for DHFSs are proposed and their properties are studied in Section 3. In Section 4, we discuss the families of GDHFCOA operators. In Section 5, we develop an approach to multiple attribute decision making problems based on GDHFCOA operator under dual hesitant fuzzy environment. An illustrative example is also given to show the effectiveness of the developed approach in Section 6. We conclude the paper and give some remarks in Section 7.

\section{Preliminaries}

Torra [21] generalized FSs to HFSs as follows.

Definition 1 (see [21]). Let $X$ be a reference set; then we define hesitant fuzzy set on $X$ in terms of a function that when applied to $X$ returns a sunset of $[0,1]$.

To be easily understood, Xia and Xu [22] express the HFS by a mathematical symbol: $E=\left(\left\langle x, h_{E}(x)\right\rangle \mid x \in X\right)$, where $h_{E}(x)$ is a set of some values in $[0,1]$, denoting the possible membership degree of the element $x \in X$ to the set $E$. For convenience, Xia and Xu [22] call $h=h_{E}(x)$ a hesitant fuzzy element (HFE) and $H$ the set of all HFEs when there is no confusion.

Nevertheless, HFS only considers the membership degree of the element $x \in X$ to the set $E$ and ignores the nonmembership degree. In order to overcome this difficulty, Zhu et al. [18] generalized HFSs to DHFS.

Definition 2 (see [18]). Let $X$ be a fixed set; then a dual hesitant fuzzy set $D$ on $X$ is defined as

$$
D=\{\langle x, h(x), g(x)\rangle \mid x \in X\}
$$

in which $h(x)$ and $g(x)$ are two sets of some values in $[0,1]$, denoting the possible membership degrees and nonmembership degrees of the element $x \in X$ to the set $D$, respectively, with conditions: $0 \leq \gamma, \eta \leq 1$ and $0 \leq \gamma^{+}+\eta^{+} \leq 1$, where $\gamma \in h(x), \eta \in g(x), \gamma^{+} \in h^{+}(x)=\cup_{\gamma \in h(x)} \max \{\gamma\}$, and $\eta^{+} \in g^{+}(x)=\cup_{\eta \in g(x)} \max \{\eta\}$ for all $x \in X$. For convenience, the pair $d(x)=\{h(x), g(x)\}$ is called a dual hesitant fuzzy element (DHFE) and denoted by $d=\{h, g\}$.

In order to compare two dual hesitant fuzzy elements, corresponding score function is defined as follows.

Definition 3 (see [18]). Let $d_{1}=\left\{h_{1}, g_{1}\right\}$ and $d_{2}=\left\{h_{2}, g_{2}\right\}$ be any two DHFEs; then the score function of $d_{i}(i=1,2)$ is

$$
S\left(d_{i}\right)=\frac{1}{n\left(h_{i}\right)} \sum_{\gamma \in h_{i}} \gamma-\frac{1}{n\left(g_{i}\right)} \sum_{\eta \in g_{i}} \eta \quad(i=1,2)
$$

and the accuracy function of $d_{i}(i=1,2)$ is

$$
P\left(d_{i}\right)=\frac{1}{n\left(h_{i}\right)} \sum_{\gamma \in h_{i}} \gamma+\frac{1}{n\left(g_{i}\right)} \sum_{\eta \in g_{i}} \eta \quad(i=1,2),
$$

where $n\left(h_{i}\right)$ and $n\left(g_{i}\right)$ are the numbers of the elements in $h_{i}$ and $g_{i}$, respectively. Then

(i) if $S\left(d_{1}\right)>S\left(d_{2}\right)$, then $d_{1}$ is superior to $d_{2}$, denoted by $d_{1}>d_{2}$

(ii) if $S\left(d_{1}\right)=S\left(d_{2}\right)$, then

(1) if $P\left(d_{1}\right)=P\left(d_{2}\right)$, then $d_{1}$ is equivalent to $d_{2}$, denoted by $d_{1} \sim d_{2}$;

(2) if $P\left(d_{1}\right)>P\left(d_{2}\right)$, then $d_{1}$ is superior to $d_{2}$, denoted by $d_{1}>d_{2}$.

Besides, some new operations on the DHFEs $d, d_{1}$, and $d_{2}$ are also introduced in [18]:

(1) $d^{\lambda}=\cup_{\gamma \in h, \eta \in g}\left\{\left\{\gamma^{\lambda}\right\},\left\{1-(1-\eta)^{\lambda}\right\}\right\}, \lambda>0$;

(2) $\lambda d=\cup_{\gamma \in h, \eta \in g}\left\{\left\{1-(1-\gamma)^{\lambda}\right\},\left\{\mu^{\lambda}\right\}\right\}, \lambda>0$;

(3) $d_{1} \oplus d_{2}=\cup_{\gamma_{1} \in h_{1}, \gamma_{2} \in h_{2}, \eta_{1} \in g_{1}, \eta_{2} \in g_{2}}\left\{\left\{\gamma_{1}+\gamma_{2}-\gamma_{1} \gamma_{2}\right\},\left\{\eta_{1} \eta_{2}\right\}\right\}$;

(4) $d_{1} \otimes d_{2}=\cup_{\gamma_{1} \in h_{1}, \gamma_{2} \in h_{2}, \eta_{1} \in g_{1}, \eta_{2} \in g_{2}}\left\{\left\{\gamma_{1} \gamma_{2}\right\},\left\{\eta_{1}+\eta_{2}-\eta_{1} \eta_{2}\right\}\right\}$.

Wang et al. [19] developed some aggregation operators for dual hesitant fuzzy information such as DHFOWA and DHFOWG operators.

Definition 4 (see [19]). Let $d_{j}(j=1,2, \ldots, n)$ be a collection of DHFEs; then we define the dual hesitant fuzzy ordered weighted average (DHFOWA) operator as follows:

$$
\operatorname{DHFOWA}_{w}\left(d_{1}, d_{2}, \ldots, d_{n}\right)=\bigoplus_{j=1}^{n}\left(w_{j} d_{\sigma(j)}\right),
$$

where $(\sigma(1), \sigma(2), \ldots, \sigma(n))$ is a permutation of $(1,2, \ldots, n)$, such that $d_{\sigma(j-1)} \geq d_{\sigma(j)}$ for all $j=2, \ldots, n$, and $w=$ $\left(w_{1}, w_{2}, \ldots, w_{n}\right)^{T}$ is the aggregation-associated weight vector such that $w_{j} \in[0,1]$, and $\sum_{j=1}^{n} w_{j}=1$.

Definition 5 (see [19]). Let $d_{j}(j=1,2, \ldots, n)$ be a collection of DHFEs; then we define the dual hesitant fuzzy ordered weighted geometric (DHFOWG) operator as follows:

$$
\operatorname{DHFOWG}_{w}\left(d_{1}, d_{2}, \ldots, d_{n}\right)=\bigotimes_{j=1}^{n}\left(d_{\sigma(j)}\right)^{w_{j}},
$$

where $(\sigma(1), \sigma(2), \ldots, \sigma(n))$ is a permutation of $(1,2, \ldots, n)$, such that $d_{\sigma(j-1)} \geq d_{\sigma(j)}$ for all $j=2, \ldots, n$, and $w=$ $\left(w_{1}, w_{2}, \ldots, w_{n}\right)^{T}$ is the aggregation-associated weight vector such that $w_{j} \in[0,1]$, and $\sum_{j=1}^{n} w_{j}=1$.

In order to weight the elements in $X$, a fuzzy measure $\mu$ is defined as follows.

Definition 6 (see [23]). A fuzzy measure $\mu$ on the set $X$ is a set function $\mu: \theta(X) \rightarrow[0,1]$ satisfying the following axioms and $\theta(X)$ is the set of all subsets of $X$ :

(1) $\mu(\phi)=0, \mu(X)=1$;

(2) $A \subseteq B$ implies $\mu(A) \leq \mu(B)$, for all $A, B \subseteq X$;

(3) $\mu(A \cup B)=\mu(A)+\mu(B)+\rho \mu(A) \mu(B)$, for all $A, B \subseteq X$ and $A \cap B=\phi$, where $\rho \in(-1, \infty)$. 
Especially, if $\rho=0$, then the condition (3) reduces to the axiom of additive measure: $\mu(A \cup B)=\mu(A)+\mu(B)$, for all $A, B \subseteq X$ and $A \cap B=\phi$.

If all the elements in $X$ are independent, then we have

$$
\mu(A)=\sum_{x_{i} \in A} \mu\left(\left\{x_{i}\right\}\right), \quad \forall A \subseteq X .
$$

The discrete Choquet integral is a linear expression up to a reordering of the elements, which is defined as below.

Definition 7 (see [24]). Let $f$ be a positive real-valued function on $X$, and let $\mu$ be a fuzzy measure on $X$. The discrete Choquet integral of $f$ with respect to $\mu$ is defined by

$$
C_{\mu}(f)=\sum_{i=1}^{n} f_{\sigma(i)}\left[\mu\left(A_{\sigma(i)}\right)-\mu\left(A_{\sigma(i-1)}\right)\right]
$$

where $(\sigma(1), \sigma(2), \ldots, \sigma(n))$ is a permutation of $(1,2, \ldots, n)$, such that $f_{\sigma(i-1)} \geq f_{\sigma(i)}$ for all $i=2,3, \ldots, n, A_{\sigma(k)}=\left\{x_{\sigma(j)} \mid\right.$ $j \leq k\}$, for $k \geq 1$, and $A_{\sigma(0)}=\phi$.

\section{Generalized Aggregation Operators for DHFS}

In this section, by introducing parameter $\lambda$, we will propose some generalized aggregation operators for DHFSs.

Definition 8. Let $d_{j}(j=1,2, \ldots, n)$ be a collection of DHFEs and let $\lambda>0$; then we define the generalized dual hesitant fuzzy weighted average (GDHFWA) operator as below:

$$
\operatorname{GDHFWA}_{w}\left(d_{1}, d_{2}, \ldots, d_{n}\right)=\left(\bigoplus_{j=1}^{n} w_{j} d_{j}^{\lambda}\right)^{1 / \lambda},
$$

where $w=\left(w_{1}, w_{2}, \ldots, w_{n}\right)^{T}$ is the weight vector of $d_{j}(j=$ $1,2, \ldots, n)$ and $w_{j}>0, \sum_{j=1}^{n} w_{j}=1$.

According to the operational laws of DHFEs, we can get the theorem below.

Theorem 9. Let $d_{j}=\left\{h_{j}, g_{j}\right\}(j=1,2, \ldots, n)$ be a collection of DHFEs; then their aggregated value by using the GDHFWA operator is also a DHFE, and

$$
\begin{aligned}
\operatorname{GDHFWA}_{w}\left(d_{1}, d_{2}, \ldots, d_{n}\right) & \left\{\bigcup_{\gamma_{j} \in h_{j}, \eta_{j} \in g_{j}}\left\{\left(1-\prod_{j=1}^{n}\left(1-\gamma_{j}^{\lambda}\right)^{w_{j}}\right)^{1 / \lambda}\right\},\right. \\
& \left.\left\{1-\left(1-\prod_{j=1}^{n}\left(1-\left(1-\eta_{j}\right)^{\lambda}\right)^{w_{j}}\right)^{1 / \lambda}\right\}\right\} .
\end{aligned}
$$

Proof. By using mathematics inductive method, we prove Theorem 9 as follows.
For $n=2$ by the operational laws of DHFEs, we can get

$$
\begin{aligned}
& d_{1}^{\lambda}=\bigcup_{\gamma_{1} \in h_{1}, \eta_{1} \in g_{1}}\left\{\left\{\gamma_{1}^{\lambda}\right\},\left\{1-\left(1-\eta_{1}\right)^{\lambda}\right\}\right\}, \\
& d_{2}^{\lambda}=\bigcup_{\gamma_{2} \in h_{2}, \eta_{2} \in g_{2}}\left\{\left\{\gamma_{2}^{\lambda}\right\},\left\{1-\left(1-\eta_{2}\right)^{\lambda}\right\}\right\}, \\
& w_{1} d_{1}^{\lambda}=\bigcup_{\gamma_{1} \in h_{1}, \eta_{1} \in g_{1}}\left\{\left\{1-\left(1-\gamma_{1}^{\lambda}\right)^{w_{1}}\right\},\left\{\left(1-\left(1-\eta_{1}\right)^{\lambda}\right)^{w_{1}}\right\}\right\} . \\
& w_{2} d_{2}^{\lambda}=\bigcup_{\gamma_{2} \in h_{2}, \eta_{2} \in g_{2}}\left\{\left\{1-\left(1-\gamma_{2}^{\lambda}\right)^{w_{2}}\right\},\left\{\left(1-\left(1-\eta_{2}\right)^{\lambda}\right)^{w_{2}}\right\}\right\} . \\
& w_{1} d_{1}^{\lambda} \oplus w_{2} d_{2}^{\lambda}=\bigcup_{\gamma_{1} \in h_{1}, \gamma_{2} \in h_{2}, \eta_{1} \in g_{1}, \eta_{2} \in g_{2}}\left\{\left\{1-\left(1-\gamma_{1}^{\lambda}\right)^{w_{1}}\left(1-\gamma_{2}^{\lambda}\right)^{w_{2}}\right\},\right. \\
& \left.\quad\left\{\left(1-\left(1-\eta_{1}\right)^{\lambda}\right)^{w_{1}}\left(1-\left(1-\eta_{2}\right)^{\lambda}\right)^{w_{2}}\right\}\right\}, \\
& \left(w_{1} d_{1}^{\lambda} \oplus w_{2} d_{2}^{\lambda}\right)^{1 / \lambda}=\bigcup_{\gamma_{1} \in h_{1}, \gamma_{2} \in h_{2}, \eta_{1} \in g_{1}, \eta_{2} \in g_{2}}\left\{\left\{\left(1-\prod_{j=1}^{2}\left(1-\gamma_{j}^{\lambda}\right)^{w_{j}}\right)^{1 / \lambda}\right\},\right. \\
& \left.\left\{1-\left(1-\prod_{j=1}^{2}\left(1-\left(1-\eta_{j}\right)^{\lambda}\right)^{w_{j}}\right)^{1 / \lambda}\right\}\right\} .
\end{aligned}
$$

If Theorem 9 holds for $n=k$, that is,

$$
\begin{aligned}
\operatorname{GDHFWA}_{w}\left(d_{1}, d_{2}, \ldots, d_{k}\right) & \left\{\bigcup_{\gamma_{j} \in h_{j}, \eta_{j} \in g_{j}}\left\{\left\{1-\prod_{j=1}^{k}\left(1-\gamma_{j}^{\lambda}\right)^{w_{j}}\right)^{1 / \lambda}\right\},\right. \\
& \left.\left\{1-\left(1-\prod_{j=1}^{k}\left(1-\left(1-\eta_{j}\right)^{\lambda}\right)^{w_{j}}\right)^{1 / \lambda}\right\}\right\},
\end{aligned}
$$

then, when $n=k+1$, by the operational laws for DHFEs, we have

$$
\begin{aligned}
& \bigoplus_{j=1}^{k+1} w_{j} d_{j}^{\lambda} \\
& =\left(\bigoplus_{j=1}^{k} w_{j} d_{j}^{\lambda}\right) \bigoplus w_{k+1} d_{k+1}^{\lambda} \\
& =\left(\bigcup _ { \gamma _ { j } \in h _ { j } , \eta _ { j } \in g _ { j } } \left\{\left\{1-\prod_{j=1}^{k}\left(1-\gamma_{j}^{\lambda}\right)^{w_{j}}\right\},\right.\right.
\end{aligned}
$$




$$
\begin{gathered}
\left.\left\{\prod_{j=1}^{k}\left(1-\left(1-\eta_{j}\right)^{\lambda}\right)^{w_{j}}\right\}\right\} \bigoplus w_{k+1} d_{k+1}^{\lambda} \\
=\bigcup_{\gamma_{j} \in h_{j}, \eta_{j} \in g_{j}}\left\{\left\{1-\prod_{j=1}^{k+1}\left(1-\gamma_{j}^{\lambda}\right)^{w_{j}}\right\},\right. \\
\left.\left\{\prod_{j=1}^{k+1}\left(1-\left(1-\eta_{j}\right)^{\lambda}\right)^{w_{j}}\right\}\right\} .
\end{gathered}
$$

Then, we get

$$
\begin{aligned}
& \left(\bigoplus_{j=1}^{k+1} w_{j} d_{j}^{\lambda}\right)^{1 / \lambda} \\
& =\bigcup_{\gamma_{j} \in h_{j}, \eta_{j} \in g_{j}}\left\{\left\{\left(1-\prod_{j=1}^{k+1}\left(1-\gamma_{j}^{\lambda}\right)^{w_{j}}\right)^{1 / \lambda}\right\},\right. \\
& \\
& \left.\left\{1-\left(1-\prod_{j=1}^{k+1}\left(1-\left(1-\eta_{j}\right)^{\lambda}\right)^{w_{j}}\right)^{1 / \lambda}\right\}\right\} .
\end{aligned}
$$

That is, Theorem 9 holds for $n=k+1$. Thus, by the principle of mathematical induction Theorem 9 holds for all $n$.

By Theorem 9, we can prove that the GDHFWA operator has the following properties.

Theorem 10 (pseudo-idempotency). Let $d_{j}=\left\{h_{j}, g_{j}\right\}(j=$ $1,2, \ldots, n)$ be a collection of DHFEs. If $\lambda=1, d_{1}=d_{2}=\cdots=$ $d_{n}=d$, and their weights $w_{1}=w_{2}=\cdots=w_{n}=1 / n$, then $\operatorname{GDHFWA}_{w}\left(d_{1}, d_{2}, \ldots, d_{n}\right) \geq d$.

Proof. Let $d_{1}=d_{2}=\cdots=d_{n}=d=\{h, g\}$, where $h=$ $\left\{\gamma_{1}, \gamma_{2}, \ldots, \gamma_{k}\right\}, g=\left\{\eta_{1}, \eta_{2}, \ldots, \eta_{s}\right\}$. By Theorem 9, we obtain

$$
\begin{aligned}
\widetilde{d} & =\operatorname{GDHFWA}_{w}\left(d_{1}, d_{2}, \ldots, d_{n}\right) \\
& =\bigcup_{\gamma_{j} \in h_{j}, \eta_{j} \in g_{j}}\left\{\left\{1-\prod_{j=1}^{n}\left(1-\gamma_{j}\right)^{1 / n}\right\},\left\{\prod_{j=1}^{n} \eta_{j}^{1 / n}\right\}\right\} .
\end{aligned}
$$

Let $\widetilde{d}=\{\widetilde{h}, \widetilde{g}\}$; then we can easily know that $\widetilde{h}$ contains $k^{n}$ numbers while $\tilde{g}$ contains $s^{n}$ numbers. By Definition 3 and multiplication of polynomial, we acquire

$$
\begin{aligned}
S(\tilde{d})= & 1-\frac{\left[\left(1-\gamma_{1}\right)^{1 / n}+\left(1-\gamma_{2}\right)^{1 / n}+\cdots\left(1-\gamma_{k}\right)^{1 / n}\right]^{n}}{k^{n}} \\
& -\frac{\left(\eta_{1}^{1 / n}+\eta_{2}^{1 / n}+\cdots+\eta_{s}^{1 / n}\right)^{n}}{s^{n}} .
\end{aligned}
$$

As we all know, $f(x)=x^{1 / n}$ is concave when $x \in[0,1]$. By Jensen's inequality, we have

$$
\begin{aligned}
& \frac{\left(1-\gamma_{1}\right)^{1 / n}+\left(1-\gamma_{2}\right)^{1 / n}+\cdots+\left(1-\gamma_{k}\right)^{1 / n}}{k} \\
& \quad \leq\left(\frac{\left(1-\gamma_{1}\right)+\left(1-\gamma_{2}\right)+\cdots+\left(1-\gamma_{k}\right)}{k}\right)^{1 / n} \\
& \quad=\left(1-\frac{\gamma_{1}+\gamma_{2}+\cdots+\gamma_{k}}{k}\right)^{1 / n},
\end{aligned}
$$

and further,

$$
\begin{gathered}
1-\frac{\left[\left(1-\gamma_{1}\right)^{1 / n}+\left(1-\gamma_{2}\right)^{1 / n}+\cdots\left(1-\gamma_{k}\right)^{1 / n}\right]^{n}}{k^{n}} \\
\geq \frac{\gamma_{1}+\gamma_{2}+\cdots+\gamma_{k}}{k} .
\end{gathered}
$$

Similarly, we have

$$
\frac{\left(\eta_{1}^{1 / n}+\eta_{2}^{1 / n}+\cdots+\eta_{s}^{1 / n}\right)^{n}}{s^{n}} \leq \frac{\eta_{1}+\eta_{2}+\cdots+\eta_{s}}{s} .
$$

So we get

$$
\begin{aligned}
S(\tilde{d})= & 1-\frac{\left[\left(1-\gamma_{1}\right)^{1 / n}+\left(1-\gamma_{2}\right)^{1 / n}+\cdots\left(1-\gamma_{k}\right)^{1 / n}\right]^{n}}{k^{n}} \\
& -\frac{\left(\eta_{1}^{1 / n}+\eta_{2}^{1 / n}+\cdots+\eta_{s}^{1 / n}\right)^{n}}{s^{n}} \\
\geq & \frac{\gamma_{1}+\gamma_{2}+\cdots+\gamma_{k}}{k}-\frac{\eta_{1}+\eta_{2}+\cdots+\eta_{s}}{s} \\
= & S(d) .
\end{aligned}
$$

This completes the proof.

Remark 11. Theorem 10 shows that idempotency of GDHFWA operator usually does not hold. When $\lambda=1$, GDHFWA degenerates to DHFWA, so Theorem 10 also indicates that the Theorem 2 in [19] is not correct. To further clarify this, we give a concrete example in the following.

Example 12. Let $\lambda=1, d_{1}=d_{2}=d_{3}=d=$ $\{\{0.6,0.7,0.8\},\{0.1,0.2\}\}$ and let $w_{1}=w_{2}=w_{3}=1 / 3$. By Theorem 9, we can compute

$$
\begin{aligned}
& \text { GDHFWA }_{w}\left(d_{1}, d_{2}, d_{3}\right) \\
& =\{\{0.6,0.636576,0.68252,0.636576,0.669807,
\end{aligned}
$$


$0.669807,0.71155,0.669807,0.7,0.737926$,

0.71155, 0.737926, 0.771057, 0.68252, 0.71155,

$0.748016,0.71155,0.737926,0.771057,0.748016$,

$0.771057,0.8\}$,

$\{0.1,0.125992,0.125992,0.15874,0.125992$,

$$
0.15874,0.15874,0.2\}\} \text {. }
$$

Then, by Definition 3, we have $S\left(\operatorname{GDHFWA}_{w}\left(d_{1}, d_{2}, d_{3}\right)\right)=$ $0.563392>0.55=S(d)$.

Theorem 13 (boundedness). Let $d_{j}=\left\{h_{j}, g_{j}\right\},(j=$ $1,2, \ldots, n)$ be a collection of DHFEs, and let

$$
\begin{gathered}
\gamma_{*}=\min \left\{\gamma \in h_{j} \mid j=1,2, \ldots, n\right\}, \\
\gamma^{*}=\max \left\{\gamma \in h_{j} \mid j=1,2, \ldots, n\right\}, \\
\eta_{*}=\min \left\{\eta \in g_{j} \mid j=1,2, \ldots, n\right\}, \\
\eta^{*}=\max \left\{\eta \in g_{j} \mid j=1,2, \ldots, n\right\}, \\
d^{-}=\left\{\gamma_{*}, \eta^{*}\right\}, d^{+}=\left\{\gamma^{*}, \eta_{*}\right\} .
\end{gathered}
$$

Then

$$
d^{-} \leq \operatorname{GDHFWA}_{w}\left(d_{1}, d_{2}, \ldots, d_{n}\right) \leq d^{+} .
$$

Proof. By Theorem 9, we get

$$
\begin{aligned}
\operatorname{GDHFWA}_{w}\left(d_{1}, d_{2}, \ldots, d_{n}\right) & \left\{\left\{\left(1-\prod_{j=1}^{n}\left(1-\gamma_{j}^{\lambda}\right)^{w_{j}}\right)^{1 / \lambda}\right\},\right. \\
\bigcup_{\gamma_{j} \in h_{j}, \eta_{j} \in g_{j}}\{ & \left.\left\{1-\left(1-\prod_{j=1}^{n}\left(1-\left(1-\eta_{j}\right)^{\lambda}\right)^{w_{j}}\right)^{1 / \lambda}\right\}\right\} .
\end{aligned}
$$

By the Definition of $\gamma_{*}, \gamma^{*}, \eta_{*}, \eta^{*}$, we know

$$
\begin{aligned}
\left(1-\prod_{j=1}^{n}\left(1-\gamma_{*}^{\lambda}\right)^{w_{j}}\right)^{1 / \lambda} & \leq\left(1-\prod_{j=1}^{n}\left(1-\gamma_{j}^{\lambda}\right)^{w_{j}}\right)^{1 / \lambda} \\
& \leq\left(1-\prod_{j=1}^{n}\left(1-\gamma^{* \lambda}\right)^{w_{j}}\right)^{1 / \lambda} .
\end{aligned}
$$

Using the condition $\sum_{j=1}^{n} w_{j}=1$, we acquire

$$
\gamma_{*} \leq\left(1-\prod_{j=1}^{n}\left(1-\gamma_{j}^{\lambda}\right)^{w_{j}}\right)^{1 / \lambda} \leq \gamma^{*} .
$$

Similarly, we obtain

$$
\eta_{*} \leq 1-\left(1-\prod_{j=1}^{n}\left(1-\left(1-\eta_{j}\right)^{\lambda}\right)^{w_{j}}\right)^{1 / \lambda} \leq \eta^{*} .
$$

Theorem 14 (quasi-monotonicity). Let $d_{j}=\left\{h_{j}, g_{j}\right\}(j=$ $1,2, \ldots, n)$ be a collection of DHEEs, where $h_{j}=$ $\left\{\gamma_{j 1}, \gamma_{j 2}, \ldots, \gamma_{j k}\right\}, g_{j}=\left\{\eta_{j 1}, \eta_{j 2}, \ldots, \eta_{j s}\right\}(j=1,2, \ldots, n)$. Let $d_{j}^{\prime}=\left\{h_{j}^{\prime}, g_{j}^{\prime}\right\}(j=1,2, \ldots, n)$ be another collection of DHEEs, where $h_{j}^{\prime}=\left\{\gamma_{j 1}^{\prime}, \gamma_{j 2}^{\prime}, \ldots, \gamma_{j k}^{\prime}\right\}, g_{j}^{\prime}=\left\{\eta_{j 1}^{\prime}, \eta_{j 2}^{\prime}, \ldots, \eta_{j s}^{\prime}\right\}(j=$ $1,2, \ldots, n)$. If $\gamma_{j p} \leq \gamma_{j p}^{\prime}, \eta_{j q} \geq \eta_{j q}^{\prime}$, for all $p=1,2, \ldots, k$, for all $q=1,2, \ldots, s$, then

$\operatorname{GDHFWA}_{w}\left(d_{1}, d_{2}, \ldots, d_{n}\right) \leq \operatorname{GDHFWA}_{w}\left(d_{1}^{\prime}, d_{2}^{\prime}, \ldots, d_{n}^{\prime}\right)$.

Proof. By Theorem 9, we get

$$
\begin{aligned}
& \operatorname{GDHFWA}_{w}\left(d_{1}, d_{2}, \ldots, d_{n}\right) \\
& =\bigcup_{\gamma_{j p} \in h_{j}, \eta_{j q} \in g_{j}}\left\{\left\{\left(1-\prod_{j=1}^{n}\left(1-\gamma_{j p}^{\lambda}\right)^{w_{j}}\right)^{1 / \lambda}\right\}\right. \text {, } \\
& \left.\left\{1-\left(1-\prod_{j=1}^{n}\left(1-\left(1-\eta_{j q}\right)^{\lambda}\right)^{w_{j}}\right)^{1 / \lambda}\right\}\right\}, \\
& \operatorname{GDHFWA}_{w}\left(d_{1}^{\prime}, d_{2}^{\prime}, \ldots, d_{n}^{\prime}\right) \\
& \begin{aligned}
=\bigcup_{\gamma_{j p}^{\prime} \in h_{j, \eta_{j q}^{\prime}}^{\prime} \in g_{j}^{\prime}}\{ & \left\{\left(1-\prod_{j=1}^{n}\left(1-\gamma_{j p}^{\prime \lambda}\right)^{w_{j}}\right)^{1 / \lambda}\right\}, \\
& \left.\left\{1-\left(1-\prod_{j=1}^{n}\left(1-\left(1-\eta_{j q}^{\prime}\right)^{\lambda}\right)^{w_{j}}\right)^{1 / \lambda}\right\}\right\} .
\end{aligned}
\end{aligned}
$$

Since $\gamma_{j p} \leq \gamma_{j p}^{\prime}, \eta_{j q} \geq \eta_{j q}^{\prime}$, for all $p=1,2, \ldots, k$, for all $q=$ $1,2, \ldots, s$, we have

$$
\begin{gathered}
\left(1-\prod_{j=1}^{n}\left(1-\gamma_{j p}^{\lambda}\right)^{w_{j}}\right)^{1 / \lambda} \leq\left(1-\prod_{j=1}^{n}\left(1-\gamma_{j p}^{\prime \lambda}\right)^{w_{j}}\right)^{1 / \lambda}, \\
1-\left(1-\prod_{j=1}^{n}\left(1-\left(1-\eta_{j q}\right)^{\lambda}\right)^{w_{j}}\right)^{1 / \lambda} \\
\geq 1-\left(1-\prod_{j=1}^{n}\left(1-\left(1-\eta_{j q}^{\prime}\right)^{\lambda}\right)^{w_{j}}\right)^{1 / \lambda} .
\end{gathered}
$$

Thus, by Definition 3, S(GDHFWA $\left.{ }_{w}\left(d_{1}, d_{2}, \ldots, d_{n}\right)\right) \leq$ $S\left(\operatorname{GDHFWA}_{w}\left(d_{1}^{\prime}, d_{2}^{\prime}, \ldots, d_{n}^{\prime}\right)\right)$.

When we need to weight the ordered positions of the dual hesitant fuzzy arguments instead of weighting the arguments themselves, GDHFWA can be generalized to GDHFOWA.

Definition 15. Let $d_{j}(j=1,2, \ldots, n)$ be a collection of DHFEs and let $\lambda>0$; then we define the generalized 
dual hesitant fuzzy ordered weighted average (GDHFOWA) operator as follows:

$$
\operatorname{GDHFOWA}_{w}\left(d_{1}, d_{2}, \ldots, d_{n}\right)=\left(\bigoplus_{j=1}^{n} w_{j} d_{\sigma(j)}^{\lambda}\right)^{1 / \lambda}
$$

where $(\sigma(1), \sigma(2), \ldots, \sigma(n))$ is a permutation of $(1,2, \ldots, n)$, such that $d_{\sigma(j-1)} \geq d_{\sigma(j)}$ for all $j=2, \ldots, n$, and $w=$ $\left(w_{1}, w_{2}, \ldots, w_{n}\right)^{T}$ is the aggregation-associated weight vector such that $w_{j} \in[0,1]$, and $\sum_{j=1}^{n} w_{j}=1$.

According to the operational laws of DHFEs, we can get the theorem below. As its proof is similar to Theorem 9, we omit it for simplicity.

Theorem 16. Let $d_{j}(j=1,2, \ldots, n)$ be a collection of DHFEs; then their aggregated value by using the GDHFWA operator is also a DHFE, and

$$
\begin{aligned}
\operatorname{GDHFOWA}_{w}\left(d_{1}, d_{2}, \ldots, d_{n}\right) & \left\{\left\{\left(1-\prod_{j=1}^{n}\left(1-\gamma_{j}^{\lambda}\right)^{w_{j}}\right)^{1 / \lambda}\right\},\right. \\
& \left.\left\{1-\left(1-\prod_{j=1}^{n}\left(1-\left(1-\eta_{j}\right)^{\lambda}\right)^{w_{j}}\right)^{1 / \lambda}\right\}\right\} .
\end{aligned}
$$

Then we will discuss some properties of GDHOWA operator, as their proofs are parallel to Theorems 10, 13, and 14 , and we need not to prove them.

Theorem 17 (pseudo-idempotency). Let $d_{j}=\left\{h_{j}, g_{j}\right\}(j=$ $1,2, \ldots, n)$ be a collection of DHFEs. If $\lambda=1, d_{1}=d_{2}=\cdots=$ $d_{n}=d$, and their weights $w_{1}=w_{2}=\cdots=w_{n}=1 / n$, then $\operatorname{GDHFOWA}_{w}\left(d_{1}, d_{2}, \ldots, d_{n}\right) \geq d$.

Theorem 18 (boundedness). Let $d_{j}=\left\{h_{j}, g_{j}\right\}(j=$ $1,2, \ldots, n)$ be a collection of DHFEs, and $d^{-}, d^{+}$are defined as before; then

$$
d^{-} \leq \operatorname{GDHFOWA}_{w}\left(d_{1}, d_{2}, \ldots, d_{n}\right) \leq d^{+} .
$$

Theorem 19 (quasi-monotonicity). Let $d_{j}=\left\{h_{j}, g_{j}\right\}(j=$ $1,2, \ldots, n)$ be a collection of DHEEs, where $h_{j}=$ $\left\{\gamma_{j 1}, \gamma_{j 2}, \ldots, \gamma_{j k}\right\}, g_{j}=\left\{\eta_{j 1}, \eta_{j 2}, \ldots, \eta_{j s}\right\}(j=1,2, \ldots, n)$. Let $d_{j}^{\prime}=\left\{h_{j}^{\prime}, g_{j}^{\prime}\right\}(j=1,2, \ldots, n)$ be another collection of DHEEs, where $h_{j}^{\prime}=\left\{\gamma_{j 1}^{\prime}, \gamma_{j 2}^{\prime}, \ldots, \gamma_{j k}^{\prime}\right\}, g_{j}^{\prime}=\left\{\eta_{j 1}^{\prime}, \eta_{j 2}^{\prime}, \ldots, \eta_{j s}^{\prime}\right\}(j=$ $1,2, \ldots, n)$. If $\gamma_{\sigma(j) p} \leq \gamma_{\sigma(j) p}^{\prime}, \eta_{\sigma(j) q} \geq \eta_{\sigma(j) q}^{\prime}($ for all $p=1,2, \ldots, k$, for all $q=1,2, \ldots, s)$, where $(\sigma(1), \sigma(2), \ldots, \sigma(n))$ is a permutation of $(1,2, \ldots, n)$, such that $d_{\sigma(j-1)} \geq d_{\sigma(j)}, d_{\sigma(j-1)}^{\prime} \geq d_{\sigma(j)}^{\prime}$ for all $j=2, \ldots, n$; then

$$
\begin{aligned}
& \operatorname{GDHFOWA}_{w}\left(d_{1}, d_{2}, \ldots, d_{n}\right) \\
& \quad \leq \operatorname{GDHFOWA}_{w}\left(d_{1}^{\prime}, d_{2}^{\prime}, \ldots, d_{n}^{\prime}\right) .
\end{aligned}
$$

By Theorem 16 and commutative law of multiplication, we can easily draw the commutativity of GDHFOWA operator.

Theorem 20 (commutativity). Let $d_{j}(j=1,2, \ldots, n)$ be a collection of DHFEs; then

$$
\begin{aligned}
& \operatorname{GDHFOWA}_{w}\left(d_{1}, d_{2}, \ldots, d_{n}\right) \\
& \quad=\operatorname{GDHFOWA}_{w}\left(d_{1}^{\prime}, d_{2}^{\prime}, \ldots, d_{n}^{\prime}\right),
\end{aligned}
$$

where $d_{j}^{\prime}(j=1,2, \ldots, n)$ is any permutation of $d_{j}(j=$ $1,2, \ldots, n)$.

Next, we will develop the dual hesitant fuzzy Choquet ordered aggregation (DHFCOA) operator and develop it to GDHFCOA.

Definition 21. Let $d_{j}(j=1,2, \ldots, n)$ be a collection of DHFEs, and let $\mu$ be a fuzzy measure on $X$; then we call

$$
\begin{aligned}
& \operatorname{DHFCOA}_{\mu}\left(d_{1}, d_{2}, \ldots, d_{n}\right) \\
& =\bigoplus_{j=1}^{n}\left(\left(\mu\left(A_{\sigma(j)}\right)-\mu\left(A_{\sigma(j-1)}\right)\right) d_{\sigma(j)}\right)
\end{aligned}
$$

the dual hesitant fuzzy Choquet ordered aggregation (DHFCOA) operator, where $(\sigma(1), \sigma(2), \ldots, \sigma(n))$ is a permutation of $(1,2, \ldots, n)$, such that $d_{\sigma(j-1)} \geq d_{\sigma(j)}$ for all $j=2,3, \ldots, n$, $A_{\sigma(k)}=\left\{x_{\sigma(j)} \mid j \leq k\right\}$, for $k \geq 1$, and $A_{\sigma(0)}=\phi$.

The DHFCOA operator can be easily transformed into the following form by induction on $n$ :

$$
\begin{aligned}
& \operatorname{DHFCOA}_{\mu}\left(d_{1}, d_{2}, \ldots, d_{n}\right) \\
& =\bigoplus_{j=1}^{n}\left(\left(\mu\left(A_{\sigma(j)}\right)-\mu\left(A_{\sigma(j-1)}\right)\right) d_{\sigma(j)}\right) \\
& =\bigcup_{\gamma_{\sigma(j)} \in h_{\sigma(j)}, \eta_{\sigma(j)} \in g_{\sigma(j)}}\left\{\left\{1-\prod_{j=1}^{n}\left(1-\gamma_{\sigma(j)}\right)^{\mu\left(A_{\sigma(j)}\right)-\mu\left(A_{\sigma(j-1)}\right)}\right\},\right. \\
& \left.\left\{\prod_{j=1}^{n}\left(\eta_{\sigma(j)}\right)^{\mu\left(A_{\sigma(j)}\right)-\mu\left(A_{\sigma(j-1)}\right)}\right\}\right\} \text {. }
\end{aligned}
$$

Obviously, this aggregated value is still a dual hesitant fuzzy element.

Particularly, if $\mu\left(\left\{x_{\sigma(j)}\right\}\right)=\mu\left(A_{\sigma(j)}\right)-\mu\left(A_{\sigma(j-1)}\right), j=$ $1,2, \ldots, n$; then DHFCOA operator degenerates into DHFWA operator. If $\mu(A)=\sum_{x_{j} \in A} \mu\left(x_{j}\right)$, for all $A \subseteq X$, let $\omega_{j}=\mu\left(A_{\sigma(j)}\right)-\mu\left(A_{\sigma(j-1)}\right), j=1,2, \ldots, n$, where $\omega=\left(\omega_{1}, \omega_{2}, \ldots, \omega_{n}\right)^{T}, \omega_{j} \geq 0, j=1,2, \ldots, n$, and $\sum_{j=1}^{n} \omega_{j}=1$; then DHFCOA operator reduces to DHFOWA operator. 
Definition 22. Let $d_{j}(j=1,2, \ldots, n)$ be a collection of DHFEs, $\lambda>0$, and let $\mu$ be a fuzzy measure on $X$; then we call

$$
\begin{aligned}
& \operatorname{GDHFCOA}_{\mu}\left(d_{1}, d_{2}, \ldots, d_{n}\right) \\
& =\left(\bigoplus_{j=1}^{n}\left(\mu\left(A_{\sigma(j)}\right)-\mu\left(A_{\sigma(j-1)}\right)\right) d_{\sigma(j)}^{\lambda}\right)^{1 / \lambda}
\end{aligned}
$$

the generalized dual hesitant fuzzy Choquet ordered aggregation (GDHFCOA) operator, where $(\sigma(1), \sigma(2), \ldots, \sigma(n))$ is a permutation of $(1,2, \ldots, n)$, such that $d_{\sigma(j-1)} \geq d_{\sigma(j)}$ for all $j=2,3, \ldots, n, A_{\sigma(k)}=\left\{x_{\sigma(j)} \mid j \leq k\right\}$, for $k \geq 1$, and $A_{\sigma(0)}=\phi$.

Theorem 23. Let $d_{j}(j=1,2, \ldots, n)$ be a collection of DHFEs; then their aggregated value by using the GDHFCOA operator is also a DHFE, and

$$
\begin{aligned}
\operatorname{GDHFCOA}_{\mu}( & \left.d_{1}, d_{2}, \ldots, d_{n}\right) \\
=\bigcup_{\gamma_{j} \in h_{\sigma(j)}, \eta_{j} \in g_{\sigma(j)}}\{ & \left\{\left(1-\prod_{j=1}^{n}\left(1-\gamma_{j}^{\lambda}\right)^{\mu\left(A_{\sigma(j)}\right)-\mu\left(A_{\sigma(j-1)}\right)}\right)^{1 / \lambda}\right\}, \\
& \left.\left\{1-\left(1-\prod_{j=1}^{n}\left(1-\left(1-\eta_{j}\right)^{\lambda}\right)^{\mu\left(A_{\sigma(j)}\right)-\mu\left(A_{\sigma(j-1)}\right)}\right)^{1 / \lambda}\right\}\right\} .
\end{aligned}
$$

Proof. By using mathematics inductive method, we prove Theorem 23 as follows.

For $n=2$, by the operational laws of DHFEs, we can get

$$
\begin{aligned}
& d_{\sigma(1)}^{\lambda}=\bigcup_{\gamma_{1} \in h_{\sigma(1)}, \eta_{1} \in g_{\sigma(1)}}\left\{\left\{\gamma_{1}^{\lambda}\right\},\left\{1-\left(1-\eta_{1}\right)^{\lambda}\right\}\right\}, \\
& d_{\sigma(2)}^{\lambda}=\bigcup_{\gamma_{2} \in h_{\sigma(2)}, \eta_{2} \in g_{\sigma(2)}}\left\{\left\{\gamma_{2}^{\lambda}\right\},\left\{1-\left(1-\eta_{2}\right)^{\lambda}\right\}\right\}, \\
& \left(\mu\left(A_{\sigma(1)}\right)-\mu\left(A_{\sigma(0)}\right)\right) d_{\sigma(1)}^{\lambda} \\
& =\bigcup_{\gamma_{1} \in h_{\sigma(1)}, \eta_{1} \in g_{\sigma(1)}}\left\{\left\{1-\left(1-\gamma_{1}^{\lambda}\right)^{\mu\left(A_{\sigma(1)}\right)-\mu\left(A_{\sigma(0)}\right)}\right\},\right. \\
& \left.\left(\mu\left(A_{\sigma(2)}\right)-\mu\left(A_{\sigma(1)}\right)\right) d_{\sigma(2)}^{\lambda}\left\{\left(1-\left(1-\eta_{1}\right)^{\lambda}\right)^{\mu\left(A_{\sigma(1)}\right)-\mu\left(A_{\sigma(0)}\right)}\right\}\right\}, \\
& =\bigcup_{\gamma_{2} \in h_{\sigma(2)}, \eta_{2} \in g_{\sigma(2)}}\left\{\left\{1-\left(1-\gamma_{2}^{\lambda}\right)^{\mu\left(A_{\sigma(2)}\right)-\mu\left(A_{\sigma(1)}\right)}\right\},\right. \\
& \left.\left\{\left(1-\left(1-\eta_{2}\right)^{\lambda}\right)^{\mu\left(A_{\sigma(2)}\right)-\mu\left(A_{\sigma(1)}\right)}\right\}\right\} .
\end{aligned}
$$

$$
\begin{aligned}
& \left(\mu\left(A_{\sigma(1)}\right)-\mu\left(A_{\sigma(0)}\right)\right) d_{\sigma(1)}^{\lambda} \oplus\left(\mu\left(A_{\sigma(2)}\right)-\mu\left(A_{\sigma(1)}\right)\right) d_{\sigma(2)}^{\lambda} \\
& =\bigcup_{\gamma_{i} \in h_{\sigma(i)}, \eta_{j} \in g_{\sigma(j)}}\left\{\left\{1-\prod_{j=1}^{2}\left(1-\gamma_{j}^{\lambda}\right)^{\left(\mu\left(A_{\sigma(j)}\right)-\mu\left(A_{\sigma(j-1)}\right)\right)}\right\},\right. \\
& \left.\left\{1-\left(1-\prod_{j=1}^{2} 1-\left(1-\eta_{j}\right)^{\lambda}\right)^{\mu\left(A_{\sigma(j)}\right)-\mu\left(A_{\sigma(j-1)}\right)}\right\}\right\}, \\
& \left(\left(\mu\left(A_{\sigma(1)}\right)-\mu\left(A_{\sigma(0)}\right)\right) d_{\sigma(1)}^{\lambda} \oplus\left(\mu\left(A_{\sigma(2)}\right)-\mu\left(A_{\sigma(1)}\right)\right) d_{\sigma(2)}^{\lambda}\right)^{1 / \lambda} \\
& =\bigcup_{\gamma_{i} \in h_{o(i)}, \eta_{j} \in g_{\sigma(j)}}\left\{\left\{\left(1-\prod_{j=1}^{2}\left(1-\gamma_{j}^{\lambda}\right)^{\left(\mu\left(A_{\sigma(j)}\right)-\mu\left(A_{\sigma(j-1)}\right)\right)}\right)^{1 / \lambda}\right\},\right. \\
& \left.\left\{1-\left(1-\prod_{j=1}^{2}\left(1-\left(1-\eta_{j}\right)^{\lambda}\right)^{\mu\left(A_{\sigma(j)}\right)-\mu\left(A_{\sigma(j-1)}\right)}\right)^{1 / \lambda}\right\}\right\}
\end{aligned}
$$

If Theorem 23 holds for $n=k$, that is,

$$
\begin{aligned}
\operatorname{GDHFCOA}_{\mu} & \left(d_{\sigma(1)}, d_{\sigma(2)}, \ldots, d_{\sigma(k)}\right) \\
=\bigcup_{\gamma_{j} \in h_{\sigma(j)} \eta_{j} \in g_{\sigma(j)}}\{ & \left\{\left(1-\prod_{j=1}^{k}\left(1-\gamma_{j}^{\lambda}\right)^{\mu\left(A_{\sigma(j)}\right)-\mu\left(A_{\sigma(j-1)}\right)}\right)^{1 / \lambda}\right\}, \\
& \left.\left\{1-\left(1-\prod_{j=1}^{k}\left(1-\left(1-\eta_{j}\right)^{\lambda}\right)^{\mu\left(A_{\sigma(j)}\right)-\mu\left(A_{\sigma(j-1)}\right)}\right)^{1 / \lambda}\right\}\right\}
\end{aligned}
$$

then, when $n=k+1$, by the operational laws for DHFEs, we have

$$
\begin{aligned}
& \bigoplus_{j=1}^{k+1}\left(\mu\left(A_{\sigma(j)}\right)-\mu\left(A_{\sigma(j-1)}\right)\right) d_{\sigma(j)}^{\lambda} \\
& =\left(\bigoplus_{j=1}^{k}\left(\mu\left(A_{\sigma(j)}\right)-\mu\left(A_{\sigma(j-1)}\right)\right) d_{\sigma(j)}^{\lambda}\right) \\
& \oplus\left(\mu\left(A_{\sigma(j+1)}\right)-\mu\left(A_{\sigma(j)}\right)\right) d_{\sigma(k+1)}^{\lambda} \\
& =\left(\bigcup_{\gamma_{j} \in h_{\sigma(j)}, \eta_{j} \in g_{\sigma(j)}}\left\{\prod_{j=1}^{k}\left(1-\gamma_{j}^{\lambda}\right)^{\mu\left(A_{\sigma(j)}\right)-\mu\left(A_{\sigma(j-1)}\right)}\right\},\right. \\
& \left.\left.\left\{\prod_{j=1}^{k}\left(1-\left(1-\eta_{j}\right)^{\lambda}\right)^{\mu\left(A_{\sigma(j)}\right)-\mu\left(A_{\sigma(j-1)}\right)}\right\}\right\}\right) \\
& \left.\oplus\left(\mu\left(A_{\sigma(j+1)}\right)-\mu\left(A_{\sigma(j)}\right)\right) d_{\sigma(k+1)}^{\lambda}\right\}
\end{aligned}
$$




$$
\begin{aligned}
=\bigcup_{j} \in h_{\sigma(j)}, \eta_{j} \in g_{\sigma(j)} & \left\{\left\{1-\prod_{j=1}^{k+1}\left(1-\gamma_{j}^{\lambda}\right)^{\mu\left(A_{\sigma(j)}\right)-\mu\left(A_{\sigma(j-1)}\right)}\right\},\right. \\
& \left.\left\{\prod_{j=1}^{k+1}\left(1-\left(1-\eta_{j}\right)^{\lambda}\right)^{\mu\left(A_{\sigma(j)}\right)-\mu\left(A_{\sigma(j-1)}\right)}\right\}\right\} .
\end{aligned}
$$

Then, we get

$$
\begin{aligned}
&\left(\bigoplus_{j=1}^{k+1}\left(\mu\left(A_{\sigma(j)}\right)-\mu\left(A_{\sigma(j-1)}\right)\right) d_{\sigma(j)}^{\lambda}\right)^{1 / \lambda}\{ \\
&=\bigcup_{\gamma_{j} \in h_{\sigma(j)}, \eta_{j} \in \mathcal{g}_{\sigma(j)}}\left\{\left(1-\prod_{j=1}^{k+1}\left(1-\gamma_{j}^{\lambda}\right)^{\mu\left(A_{\sigma(j)}\right)-\mu\left(A_{\sigma(j-1)}\right)}\right)^{1 / \lambda}\right\}, \\
&\left.\left\{1-\left(1-\prod_{j=1}^{k+1}\left(1-\left(1-\eta_{j}\right)^{\lambda}\right)^{\mu\left(A_{\sigma(j)}\right)-\mu\left(A_{\sigma(j-1)}\right)}\right)^{1 / \lambda}\right\}\right\} .
\end{aligned}
$$

That is, Theorem 23 holds for $n=k+1$. Thus, by the principle of mathematical induction, Theorem 23 holds for all $n$.

Parallel to Theorems 10, 13, 14, and 20, we can prove the similar properties of GDHFCOA operator as follows.

Theorem 24 (pseudo-idempotency). Let $d_{j}=\left\{h_{j}, g_{j}\right\}(j=$ $1,2, \ldots, n)$ be a collection of DHFEs. If $\lambda=1, d_{1}=d_{2}=\cdots=$ $d_{n}=d$, and $\mu\left(A_{\sigma(j)}\right)-\mu\left(A_{\sigma(j-1)}\right)=1 / n, j=1,2, \ldots, n$, then $\operatorname{GDHFCOA}_{\mu}\left(d_{1}, d_{2}, \ldots, d_{n}\right) \geq d$.

Theorem 25 (boundedness). Let $d_{j}=\left\{h_{j}, g_{j}\right\}(j=1,2$, $\ldots, n)$ be a collection of DHFEs, and $d^{-}, d^{+}$are defined as before; then

$$
d^{-} \leq \operatorname{GDHFCOA}\left(d_{1}, d_{2}, \ldots, d_{n}\right) \leq d^{+} .
$$

Theorem 26 (quasi-monotonicity). Let $d_{j}=\left\{h_{j}, g_{j}\right\}(j=$ $1,2, \ldots, n)$ be a collection of DHEEs, where $h_{j}=$ $\left\{\gamma_{j 1}, \gamma_{j 2}, \ldots, \gamma_{j k}\right\}, g_{j}=\left\{\eta_{j 1}, \eta_{j 2}, \ldots, \eta_{j s}\right\}(j=1,2, \ldots, n)$. Let $d_{j}^{\prime}=\left\{h_{j}^{\prime}, g_{j}^{\prime}\right\}(j=1,2, \ldots, n)$ be another collection of DHEEs, where $h_{j}^{\prime}=\left\{\gamma_{j 1}^{\prime}, \gamma_{j 2}^{\prime}, \ldots, \gamma_{j k}^{\prime}\right\}, g_{j}^{\prime}=\left\{\eta_{j 1}^{\prime}, \eta_{j 2}^{\prime}, \ldots, \eta_{j s}^{\prime}\right\}(j=$ $1,2, \ldots, n)$. If $\gamma_{\sigma(j) p} \leq \gamma_{\sigma(j) p}^{\prime}, \eta_{\sigma(j) q} \geq \eta_{\sigma(j) q}^{\prime}($ for all $p=1,2, \ldots, k$, for all $q=1,2, \ldots, s)$, where $(\sigma(1), \sigma(2), \ldots, \sigma(n))$ is a permutation of $(1,2, \ldots, n)$, such that $d_{\sigma(j-1)} \geq d_{\sigma(j)}, d_{\sigma(j-1)}^{\prime} \geq d_{\sigma(j)}^{\prime}$ for all $j=2, \ldots, n$, then

$$
\begin{aligned}
& \operatorname{GDHFCOA}_{\mu}\left(d_{1}, d_{2}, \ldots, d_{n}\right) \\
& \quad \leq \operatorname{GDHFCOA}_{\mu}\left(d_{1}^{\prime}, d_{2}^{\prime}, \ldots, d_{n}^{\prime}\right) .
\end{aligned}
$$

Theorem 27 (commutativity). Let $d_{j}(j=1,2, \ldots, n)$ be a collection of DHFEs; then

$$
\begin{aligned}
& \operatorname{GDHFCOA}_{\mu}\left(d_{1}, d_{2}, \ldots, d_{n}\right) \\
& \quad=\operatorname{GDHFCOA}_{\mu}\left(d_{1}^{\prime}, d_{2}^{\prime}, \ldots, d_{n}^{\prime}\right),
\end{aligned}
$$

where $d_{j}^{\prime}(j=1,2, \ldots, n)$ is any permutation of $d_{j}(j=$ $1,2, \ldots, n)$.

\section{Families of GDHFCOA Operators}

In this section, different types of GDHFCOA operators are considered. When we change the parameters, GDHFCOA operator can be reduced to various operators.

Theorem 28. Let $d_{j}(j=1,2, \ldots, n)$ be a collection of DHFEs, $\lambda>0$, and let $\mu$ be a fuzzy measure on $X$. If we denote $w_{j}=\mu\left(A_{\sigma(j)}\right)-\mu\left(A_{\sigma(j-1)}\right), j=1,2, \ldots, n$, then $w_{j} \in$ $[0,1], \sum_{j=1}^{n} w_{j}=1$ and GDHFCOA operator is reduced to

$$
\operatorname{GDHFOWA}_{w}\left(d_{1}, d_{2}, \ldots, d_{n}\right)=\left(\bigoplus_{j=1}^{n} w_{j} d_{\sigma(j)}^{\lambda}\right)^{1 / \lambda} .
$$

Theorem 29. Let $d_{j}(j=1,2, \ldots, n)$ be a collection of DHFEs, $\lambda>0$, and let $\mu$ be a fuzzy measure on $X$. We denote $w_{j}=$ $\mu\left(A_{\sigma(j)}\right)-\mu\left(A_{\sigma(j-1)}\right), j=1,2, \ldots, n$. If $d_{1}>d_{2}>\cdots>d_{n}$, then the GDHFOWA operator is reduced to

$$
\operatorname{GDHFWA}_{w}\left(d_{1}, d_{2}, \ldots, d_{n}\right)=\left(\bigoplus_{j=1}^{n} w_{j} d_{j}^{\lambda}\right)^{1 / \lambda}
$$

Theorem 30. Let $d_{j}(j=1,2, \ldots, n)$ be a collection of DHFEs and let $\mu$ be a fuzzy measure on $X$. We denote $w_{j}=\mu\left(A_{\sigma(j)}\right)-$ $\mu\left(A_{\sigma(j-1)}\right), j=1,2, \ldots, n$. If $\lambda=1$, then the GDHFOWA operator and GDHFWA operator are reduced to DHFOWA operator and DHFWA operator, respectively.

Theorem 31. Let $d_{j}(j=1,2, \ldots, n)$ be a collection of DHFEs, $\lambda>0$, and let $w=\left(w_{1}, w_{2}, \ldots, w_{n}\right)^{T}$ be the aggregationassociated weight vector such that $w_{j} \in[0,1]$, and $\sum_{j=1}^{n} w_{j}=$ 1.

(1) If $w=(1,0, \ldots, 0)^{T}$, then the GDHFOWA operator is reduced to the following:

$$
\operatorname{GDHFOWA}_{w}\left(d_{1}, d_{2}, \ldots, d_{n}\right)=d_{\sigma(1)}=\max _{j}\left\{d_{j}\right\} \text {. }
$$

(2) If $w=(0,0, \ldots, 1)^{T}$, then the GDHFOWA operator is reduced to the following:

$$
\operatorname{GDHFOWA}_{w}\left(d_{1}, d_{2}, \ldots, d_{n}\right)=d_{\sigma(n)}=\min _{j}\left\{d_{j}\right\} \text {. }
$$

\section{An Approach to Multiple Attribute Decision Making with Dual Hesitant Fuzzy Information}

In this section, we will utilize the GDHFCOA operator to multiple attribute decision making under dual hesitant fuzzy environment. The following assumptions or notations are used to represent the MADM problems for evaluation of theses with dual hesitant fuzzy information. Let $A=$ $\left\{A_{1}, A_{2}, \ldots, A_{m}\right\}$ be a discrete set of alternatives, and let 
$G=\left\{G_{1}, G_{2}, \ldots, G_{n}\right\}$ be the state of nature. If the decision makers provide several values for the alternative $A_{i}$ under the attribute $G_{j}$ with anonymity, these values can be considered as a dual hesitant fuzzy element $d_{i j}=\left\{h_{i j}, g_{i j}\right\}$. Suppose that the decision matrix $D=\left(d_{i j}\right)_{m \times n}$ is the dual hesitant fuzzy decision matrix, where $d_{i j}=\left\{h_{i j}, g_{i j}\right\},(i=1,2, \ldots, m$, $j=1,2, \ldots, n)$ are in the form of DHFEs. In the following, we apply the GDHFCOA operator to the multiple attribute decision making problems for evaluation of theses with dual hesitant fuzzy information.

Step 1. Confirm the fuzzy measures $\mu$ of attributes of $G$ and attributes sets of $G$.

Step 2. Utilize the decision information given in matrix $D$ and the GDHFCOA operator

$$
\begin{aligned}
& \tilde{d}_{i}= \operatorname{GDHFCOA}_{\mu}\left(d_{1}, d_{2}, \ldots, d_{n}\right) \\
&=\left(\bigoplus_{j=1}^{n}\left(\mu\left(A_{\sigma(j)}\right)-\mu\left(A_{\sigma(j-1)}\right)\right) d_{\sigma(j)}^{\lambda}\right)^{1 / \lambda} \\
&=\bigcup_{\gamma_{j} \in h_{\sigma(j)}, \eta_{j} \in g_{\sigma(j)}}\{\left\{\left(1-\prod_{j=1}^{n}\left(1-\gamma_{j}^{\lambda}\right)^{\mu\left(A_{\sigma(j)}\right)-\mu\left(A_{\sigma(j-1)}\right)}\right)^{1 / \lambda}\right\}, \\
&\left.\left\{1-\left(1-\prod_{j=1}^{n}\left(1-\left(1-\eta_{j}\right)^{\lambda}\right)^{\mu\left(A_{\sigma(j)}\right)-\mu\left(A_{\sigma(j-1)}\right)}\right)^{1 / \lambda}\right\}\right\}
\end{aligned}
$$

to derive the overall preference values $\widetilde{d}_{i}(i=1,2, \ldots, m)$ of the alternative $A_{i}$.

Step 3. Calculate the scores $s\left(\widetilde{d}_{i}\right)(i=1,2, \ldots, m)$ of the overall dual hesitant fuzzy values $\widetilde{d}_{i}(i=1,2, \ldots, m)$ by Definition 3.

Step 4. Rank all the alternatives $A_{i}(i=1,2, \ldots, m)$ in accordance with the scores $s\left(\widetilde{d}_{i}\right)(i=1,2, \ldots, m)$ and select the best one(s).

Step 5. End.

Remark 32. The advantages of the generalized dual hesitant fuzzy Choquet ordered aggregation (GDHFCOA) operator lie in four aspects.

First, there is a fuzzy measure $\mu$ in the GDHFCOA operator, which can be regarded as an extension of the weight vector. Sometimes, some attributes may have little importance, respectively, but when they gather together, they become very important. In order to deal with this situation, we can use the fuzzy measure to define a weight on not only each attribute but also each combination of attributes.

Second, our method does not assume the independence of one attribute from another and it can deal with the situation where the attributes are correlative. Traditional additive aggregation operators, such as DHFWA operator and DHFOWA operator, are all based on the assumption that the attributes are independent, and each attribute is given a fixed weight representing its importance during the decision process. Nevertheless, they do not consider the addition of the importance of individual attribute, and as a result, they cannot get reasonable results when the attributes are correlative. In real decision problems, since there are often interdependent or interactive phenomena among attributes, the overall importance of an attribute is not only determined by itself, but also by other attributes. So our method is a good choice to solve the real decision making problems.

Third, the GDHFCOA operator can accommodate situations in which the input arguments are dual hesitant fuzzy information. As dual hesitant fuzzy set is a comprehensive set containing FSs, IFSs, FMSs, and HFSs as special cases, our method can be widely used.

Fourth, the GDHFCOA operator has an additional parameter $\lambda$ which controls the power. If the parameter takes different values, the proposed operators can be evolved into many special aggregation operators, which make decision making more flexible and can meet the different needs of different decision makers. That is to say, the decision makers can choose the value of the parameter according to their preferences and interests.

\section{Numerical Example}

Thus, in this section, we will present a numerical example (adapted from $[19,25]$ ) to show evaluation of theses with dual hesitant fuzzy information in order to illustrate the proposed method. There are five theses $A_{i}(i=1,2,3,4,5)$, and we want to select the best one. Four attributes are selected by experts to evaluate the theses: (1) $G_{1}$ is the language of a thesis; (2) $G_{2}$ is the innovation; (3) $G_{3}$ is the rigor; (4) $G_{4}$ is the structure of the thesis. In order to avoid influencing each other, the experts are required to evaluate the five theses $A_{i}(i=1,2,3,4,5)$ under the above four attributes in anonymity and the decision matrix $D=\left(d_{i j}\right)_{5 \times 4}$ is presented in Table 1, where $d_{i j}=\left\{h_{i j}, g_{i j}\right\},(i=1,2,3,4,5, j=1,2,3,4)$ are in the form of DHFEs. The fuzzy measure of attribute $G_{j}(j=1,2, \ldots, 4)$ and attribute sets of $G$ are as follows: $\mu\left(G_{1}\right)=0.30, \mu\left(G_{2}\right)=0.35, \mu\left(G_{3}\right)=0.30, \mu\left(G_{4}\right)=0.22$, $\mu\left(G_{1}, G_{2}\right)=0.70, \mu\left(G_{1}, G_{3}\right)=0.60, \mu\left(G_{1}, G_{4}\right)=0.55$, $\mu\left(G_{2}, G_{3}\right)=0.50, \mu\left(G_{2}, G_{4}\right)=0.45, \mu\left(G_{3}, G_{4}\right)=0.40$, $\mu\left(G_{1}, G_{2}, G_{3}\right)=0.82, \mu\left(G_{1}, G_{2}, G_{4}\right)=0.87, \mu\left(G_{1}, G_{3}, G_{4}\right)=$ $0.75, \mu\left(G_{2}, G_{3}, G_{4}\right)=0.60, \mu\left(G_{1}, G_{2}, G_{3}, G_{4}\right)=1.00$.

6.1. The Decision Making Steps. Next, we apply the developed approach to evaluate these theses with dual hesitant fuzzy information.

Step 1. We use the decision information given in matrix $D$ and the GDHFCOA operator to obtain the overall preference values $\widetilde{d}_{i}$ of the thesis $A_{i}(i=1,2,3,4,5)$. Take thesis $A_{1}$, for example, we have (take $\lambda=1$ )

$$
\begin{aligned}
\widetilde{d}_{1} & =\operatorname{GDHFCOA}_{\mu}\left(d_{11}, d_{12}, d_{13}, d_{14}\right) \\
& =\bigcup_{\gamma_{j} \in h_{\sigma(j)}, \eta_{j} \in g_{\sigma(j)}}\left\{\left\{\left(1-\prod_{j=1}^{4}\left(1-\gamma_{j}^{\lambda}\right)^{\mu\left(A_{\sigma(j)}\right)-\mu\left(A_{\sigma(j-1)}\right)}\right)^{1 / \lambda}\right\},\right.
\end{aligned}
$$


TABLE 1: Dual hesitant fuzzy decision matrix $\widetilde{D}$.

\begin{tabular}{lcccc}
\hline & $G_{1}$ & $G_{2}$ & $G_{3}$ & $G_{4}$ \\
\hline$A_{1}$ & $\{\{0.4,0.5\},\{0.5\}\}$ & $\{\{0.5,0.6\},\{0.2,0.3\}\}$ & $\{\{0.3,0.4\},\{0.5\}\}$ & $\{\{0.4,0.6\},\{0.2,0.3\}\}$ \\
$A_{2}$ & $\{\{0.5,0.6\},\{0.1,0.3\}\}$ & $\{\{0.2,0.4,0.5\},\{0.4\}\}$ & $\{\{0.2\},\{0.6,0.7,0.8\}\}$ & $\{0.5\},\{0.3,0.4\}\}$ \\
$A_{3}$ & $\{\{0.5,0.8\},\{0.2\}\}$ & $\{\{0.1\},\{0.7,0.8\}\}$ & $\{\{0.2,0.3,0.4\},\{0.6\}\}$ & $\{\{0.4,0.6,0.7\},\{0.3\}\}$ \\
$A_{4}$ & $\{\{0.5\},\{0.4\}\}$ & $\{\{0.4,0.6,0.8\},\{0.2\}\}$ & $\{\{0.1,0.2\},\{0.6\}\}$ & $\{\{0.4\},\{0.5,0.6\}\}$ \\
$A_{5}$ & $\{\{0.4,0.6\},\{0.2\}\}$ & $\{\{0.2,0.3,0.4\},\{0.6\}\}$ & $\{\{0.4,0.5\},\{0.2\}\}$ & \\
\hline
\end{tabular}

$$
\left.\left\{1-\left(1-\prod_{j=1}^{4}\left(1-\left(1-\eta_{j}\right)^{\lambda}\right)^{\mu\left(A_{\sigma(j)}\right)-\mu\left(A_{\sigma(j-1)}\right)}\right)^{1 / \lambda}\right\}\right\} .
$$

$=\{\{0.425697,0.437091,0.468033,0.478587,0.448517$,

0.459459, 0.489171, 0.499306, 0.468844, 0.479382,

0.507999, 0.51776, 0.489949, 0.500069, 0.527549,

$0.536922\},\{0.331053,0.344751,0.38153,0.397318\}\}$.

When assigning different values to the parameter $\lambda$, we can obtain different dual hesitant fuzzy values.

Step 2. Calculate the scores $s\left(\widetilde{d}_{i}\right)(i=1,2,3,4,5)$ of the overall dual hesitant fuzzy values $\widetilde{d}_{i}(i=1,2,3,4,5)$ of the thesis $A_{i}$. Please see Table 2 .

Step 3. According to the scores $s\left(\tilde{d}_{i}\right)$ of the overall dual hesitant fuzzy values $\widetilde{d}_{i}(i=1,2, \ldots, 5)$, we rank all the theses $A_{i}(i=1,2,3,4,5)$ by comparing laws of DHFEs. The results are shown in Table 2 .

6.2. Discussion. From Table 2, we find that the values obtained by the GDHFCOA operator become bigger as the parameter $\lambda$ increases. Moreover, the rankings are different when we choose different values of $\lambda$. The trends are shown in Figure 1 as the parameter values range from 0 to 4 . We denote the score $s\left(\tilde{d}_{i}\right)$ as $Y_{i}(i=1,2, \ldots, 5)$ for short in Figure 1. From Figure 1, it can be clearly seen that

(1) when $\lambda \in(0,0.947173]$, the ranking of the five theses is $A_{4}>A_{1}>A_{2}>A_{5}>A_{3}$;

(2) when $\lambda \in(0.947173,1.14939]$, the ranking of the five theses is $A_{4}>A_{2}>A_{1}>A_{5}>A_{3}$;

(3) when $\lambda \in(1.14939,1.47194]$, the ranking of the five theses is $A_{4}>A_{2}>A_{1}>A_{3}>A_{5}$;

(4) when $\lambda \in(1.47194,1.64355]$, the ranking of the five theses is $A_{4}>A_{2}>A_{3}>A_{1}>A_{5}$;

(5) when $\lambda \in(1.64355,2.43027]$, the ranking of the five theses is $A_{4}>A_{3}>A_{2}>A_{1}>A_{5}$;

(6) when $\lambda \in(2.43027,2.85024]$, the ranking of the five theses is $A_{4}>A_{3}>A_{2}>A_{5}>A_{1}$;

(7) when $\lambda \in(2.85024,4]$, the ranking of the five theses is $A_{3}>A_{4}>A_{2}>A_{5}>A_{1}$.

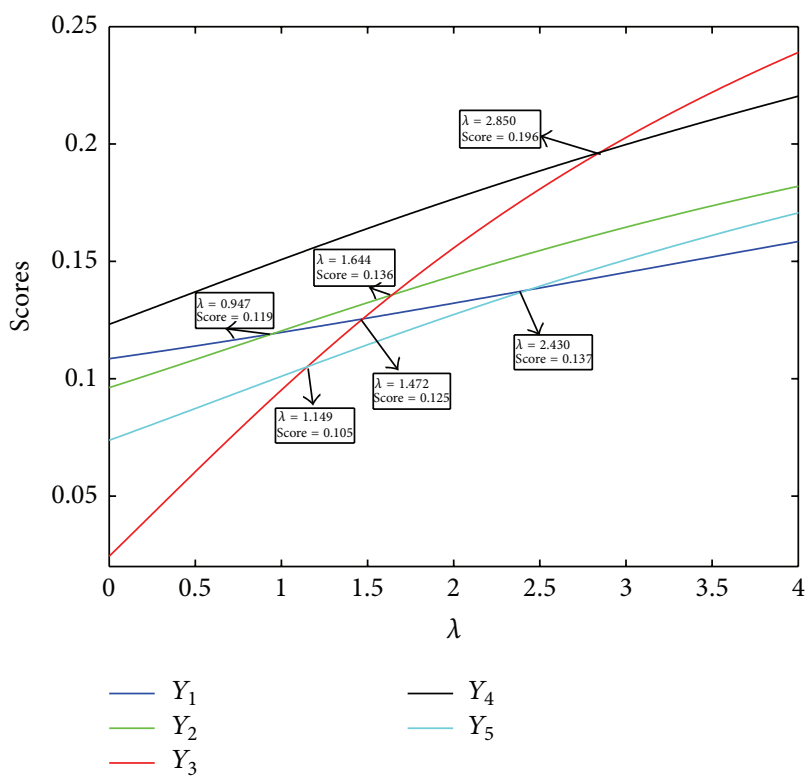

Figure 1: Scores for theses obtained by GDHFCOA operator.

Obviously, the decision makers can choose different parameters according to their interests, which make the decision more flexible.

6.3. Comparative Analysis. In order to show the merit of the proposed method, we utilized some existing methods to solve this numerical example. As the GDHFCOA operator is an ordered weighted operator, we choose DHFOWA and DHFOWG rather than DHFWA and DHFWG. Then, we utilized the method proposed by Wang et al. [19] to solve this illustrative example. For simplicity, we omit the calculation process and only list the results in Tables 3 and 4 .

From Table 2-Table 4, we can compare these methods as follows.

(1) During the calculation, we can find that the weight vectors can be obtained by the source decision information in our method. As a result, different decision data will acquire different weight vectors automatically. However, for DHFOWA operator and DHFOWG operator, the weight vectors must be given by experts in advance. Thus, the proposed method is more objective and reasonable than Wang's method [19]. 
TABLE 2: Scores of the dual hesitant fuzzy values obtained by GDHFCOA operator.

\begin{tabular}{|c|c|c|c|c|c|c|}
\hline & $s\left(\widetilde{d}_{1}\right)$ & $s\left(\widetilde{d}_{2}\right)$ & $s\left(\widetilde{d}_{3}\right)$ & $s\left(\widetilde{d}_{4}\right)$ & $s\left(\widetilde{d}_{5}\right)$ & Ranking \\
\hline$\lambda=0.5$ & 0.113941 & 0.108192 & 0.060358 & 0.13701 & 0.0873121 & $A_{4}>A_{1}>A_{2}>A_{5}>A_{3}$ \\
\hline$\lambda=1$ & 0.119733 & 0.120391 & 0.0951852 & 0.150743 & 0.101029 & $A_{4}>A_{2}>A_{1}>A_{5}>A_{3}$ \\
\hline$\lambda=1.5$ & 0.125836 & 0.132388 & 0.127193 & 0.164 & 0.11447 & $A_{4}>A_{2}>A_{3}>A_{1}>A_{5}$ \\
\hline$\lambda=2$ & 0.132178 & 0.143864 & 0.155721 & 0.176611 & 0.127319 & $A_{4}>A_{3}>A_{2}>A_{1}>A_{5}$ \\
\hline$\lambda=2.5$ & 0.138683 & 0.154615 & 0.180793 & 0.188531 & 0.139405 & $A_{4}>A_{3}>A_{2}>A_{5}>A_{1}$ \\
\hline$\lambda=3$ & 0.145275 & 0.16455 & 0.202751 & 0.199774 & 0.150659 & $A_{3}>A_{4}>A_{2}>A_{5}>A_{1}$ \\
\hline
\end{tabular}

TABLE 3: Scores for theses obtained by DHFOWA operator.

\begin{tabular}{|c|c|c|c|c|c|c|}
\hline Weight vectors & $s\left(\widetilde{d}_{1}\right)$ & $s\left(\widetilde{d}_{2}\right)$ & $s\left(\widetilde{d}_{3}\right)$ & $s\left(\widetilde{d}_{4}\right)$ & $s\left(\widetilde{d}_{5}\right)$ & Ranking \\
\hline$w=(0.35,0.1,0.42,0.13)^{T}$ & 0.119733 & 0.115672 & 0.0818534 & 0.098652 & 0.0279722 & $A_{1}>A_{2}>A_{4}>A_{3}>A_{5}$ \\
\hline$w=(0.3,0.25,0.32,0.13)^{T}$ & 0.147745 & 0.120391 & 0.120942 & 0.100435 & 0.077588 & $A_{1}>A_{3}>A_{2}>A_{4}>A_{5}$ \\
\hline$w=(0.25,0.25,0.25,0.25)^{T}$ & 0.119935 & 0.0584848 & 0.054057 & 0.0421476 & 0.0458413 & $A_{1}>A_{2}>A_{3}>A_{5}>A_{4}$ \\
\hline$w=(0.3,0.3,0.22,0.18)^{T}$ & 0.157777 & 0.113094 & 0.135685 & 0.100415 & 0.101029 & $A_{1}>A_{3}>A_{2}>A_{5}>A_{4}$ \\
\hline
\end{tabular}

TABLE 4: Scores for theses obtained by DHFOWG operator.

\begin{tabular}{lcccccc}
\hline Weight vector & $s\left(\widetilde{d}_{1}\right)$ & $s\left(\widetilde{d}_{2}\right)$ & $s\left(\widetilde{d}_{3}\right)$ & $s\left(\widetilde{d}_{4}\right)$ & $s\left(\widetilde{d}_{5}\right)$ & Ranking \\
\hline$w=(0.35,0.1,0.42,0.13)^{T}$ & 0.0692636 & 0.00329123 & -0.13716 & -0.0344067 & -0.0970861 & $A_{1}>A_{2}>A_{4}>A_{5}>A_{3}$ \\
$w=(0.3,0.25,0.32,0.13)^{T}$ & 0.0958579 & 0.0129617 & -0.0911802 & -0.0205423 & -0.0447992 & $A_{1}>A_{2}>A_{4}>A_{5}>A_{3}$ \\
$w=(0.25,0.25,0.25,0.25)^{T}$ & 0.0645639 & -0.0802401 & -0.19675 & -0.0988189 & -0.0822588 & $A_{1}>A_{2}>A_{5}>A_{4}>A_{3}$ \\
$w=(0.3,0.3,0.22,0.18)^{T}$ & 0.104939 & -0.0109346 & -0.0973555 & -0.0317627 & -0.0200345 & $A_{1}>A_{2}>A_{5}>A_{4}>A_{3}$ \\
\hline
\end{tabular}

(2) Tables 2 and 3 have some common scores such as $S\left(\widetilde{d}_{1}\right)=0.119733$. This indicates that DHFOWA operator is a special case of GDHFCOA operator under certain conditions, which has been pointed out in Theorem 30.

(3) We find that the rankings in Table 2 are quite different from Tables 3 and 4 . The reason may be that there are interdependent or interactive phenomena among attributes in this numerical example. From another perspective,

$$
\begin{aligned}
\mu\left(G_{1}\right) & +\mu\left(G_{2}\right)+\mu\left(G_{3}\right)+\mu\left(G_{4}\right) \\
= & 0.30+0.35+0.30+0.22>1 \\
= & \mu\left(G_{1}, G_{2}, G_{3}, G_{4}\right)
\end{aligned}
$$

also tells us that the attributes are correlative. The GDHFCOA operator can perform aggregation of attributes when they are correlative. However, DHFOWA and DHFOWG operators always suppose that the attributes are independent, and each attribute is given a fixed weight subjectively. So the GDHFCOA operator is a better choice here.

(4) When we change the parameter $\lambda$, we get different rankings in Table 2. This indicates that the GDHFCOA operator have an additional parameter $\lambda$, which makes decision making more flexible and can meet the needs of different types of decision makers.

\section{Conclusion}

In this paper, we have investigated the multiple attribute decision making (MADM) problem based on the GDHFCOA operator with dual hesitant fuzzy information. Firstly, some operational laws of dual hesitant fuzzy elements and score function of dual hesitant fuzzy elements as well as existing aggregation operators have been introduced. Then, motivated by the ideal of Choquet integral, the generalized dual hesitant fuzzy Choquet ordered aggregation (GDHFCOA) operator has been developed. Its advantage is that it can consider the importance of the attributes as well as the correlation among the attributes, which makes it more feasible and practical. At the same time, we have introduced several generalized aggregation operators for DHFS such as GDHFWA, and discussed their basic properties. As different parameters can be chosen in these generalized aggregation operators, the decision becomes more flexible. Furthermore, we have discussed the families of GDHFCOA operator. Next, we have applied the GDHFCOA operator to multiple attribute decision making problems with dual hesitant fuzzy information. Finally, an illustrative example for evaluation of theses has been given to demonstrate its practicality and effectiveness. In the future, we will consider the monotonicity of GDHFCOA operator and apply the dual hesitant fuzzy multiple attribute decision making to other domains.

\section{Conflict of Interests}

The authors declare that there is no conflict of interests regarding the publication of this paper. 


\section{Acknowledgments}

The authors would like to thank the editor and the anonymous referees for their valuable comments, which have been very helpful in improving the paper. This work was supported by the National Natural Science Foundation of China (no. 11371130), Research Fund for the Doctoral Program of Higher Education of China (no. 20120161110017), and China Postdoctoral Science Foundation (no. 2012M521515).

\section{References}

[1] L. A. Zadeh, "Fuzzy sets," Information and Computation, vol. 8, pp. 338-353, 1965.

[2] V. Derhami, "Similarity of learned helplessness in human being and fuzzy reinforcement learning algorithms," Journal of Intelligent and Fuzzy Systems, vol. 24, pp. 347-354, 2013.

[3] M. H. Khooban and M. R. Soltanpour, "Swarm optimization tuned fuzzy sliding mode control design for a class of nonlinear systems in presence of uncertainties," Journal of Intelligent \& Fuzzy Systems, vol. 24, no. 2, pp. 383-394, 2013.

[4] W. C. Lee and J. W. Wu, "Reply to 'open problemof fuzzy confidence interval for fuzzy process capability index,"' Journal of Intelligent and Fuzzy Systems, vol. 24, no. 1, pp. 1-3, 2013.

[5] S. Mathew and M. S. Sunitha, "Cycle connectivity in fuzzy graphs," Journal of Intelligent \& Fuzzy Systems, vol. 24, no. 3, pp. 549-554, 2013.

[6] A. F. Meyabadi and M. Ehsan, "Aheuristic fuzzy decision-based solving of redispatching problem for congestion management in restructured power systems," Journal of Intelligent and Fuzzy Systems, vol. 24, pp. 563-574, 2013.

[7] L. A. Zadeh, "The concept of a linguistic variable and its application to approximate reasoning, Part I," Information Sciences, vol. 8, pp. 199-249, 1975.

[8] S. Miyamoto, "Multisets and fuzzy multisets," in Soft Computing and Human-Centered Machines, Z. Q. Liu and S. Miyamoto, Eds., pp. 9-33, Springer, Berlin, Germany, 2000.

[9] S. Miyamoto, "Remarks on basics of fuzzy sets and fuzzy multisets," Fuzzy Sets and Systems, vol. 156, no. 3, pp. 427-431, 2005.

[10] K. T. Atanassov, "Intuitionistic fuzzy sets," Fuzzy Sets and Systems, vol. 20, no. 1, pp. 87-96, 1986.

[11] K. Atanassov and G. Gargov, "Interval valued intuitionistic fuzzy sets," Fuzzy Sets and Systems, vol. 31, no. 3, pp. 343-349, 1989.

[12] Z. Xu, "A method based on distance measure for intervalvalued intuitionistic fuzzy group decision making," Information Sciences, vol. 180, no. 1, pp. 181-190, 2010.

[13] Z. S. Xu and X. L. Zhang, "Hesitant fuzzy multi-attribute decision making based on TOPSIS with incomplete weight information," Knowledge-Based Systems, vol. 52, pp. 53-64, 2013.

[14] Z. Xu and M. Xia, "On distance and correlation measures of hesitant fuzzy information," International Journal of Intelligent Systems, vol. 26, no. 5, pp. 410-425, 2011.

[15] D. J. Yu, W. Y. Zhang, and Y. J. Xu, "Group decision making under hesitant fuzzy environment with application to personnel evaluation," Knowledge-Based Systems, vol. 52, pp. 1-10, 2013.

[16] G. Wei, "Hesitant fuzzy prioritized operators and their application to multiple attribute decision making," Knowledge-Based Systems, vol. 31, pp. 176-182, 2012.
[17] M. M. Xia, Z. S. Xu, and N. Chen, "Some hesitant fuzzy aggregation operators with their application in group decision making," Group Decision and Negotiation, vol. 22, pp. 259-279, 2013.

[18] B. Zhu, Z. Xu, and M. Xia, "Dual hesitant fuzzy sets," Journal of Applied Mathematics, vol. 2012, Article ID 879629, 13 pages, 2012.

[19] H. J. Wang, X. F. Zhao, and G. W. Wei, "Dual hesitant fuzzy aggregation operators in multiple attribute decision making," Journal of Intelligent and Fuzzy Systems, vol. 26, pp. 2281-2290, 2014.

[20] G. Choquet, “Theory of capacities," Annales de l'Institut Fourier, vol. 5, pp. 131-295, 1953.

[21] V. Torra, "Hesitant fuzzy sets," International Journal of Intelligent Systems, vol. 25, no. 6, pp. 529-539, 2010.

[22] M. Xia and Z. Xu, "Hesitant fuzzy information aggregation in decision making," International Journal of Approximate Reasoning, vol. 52, no. 3, pp. 395-407, 2011.

[23] M. Grabisch, T. Murofushi, and M. Sugeno, Fuzzy Measures and Integrals, vol. 40 of Studies in Fuzziness and Soft Computing, Physica, New York, NY, USA, 2000.

[24] Z. Y. Wang and G. J. Klir, Fuzzy Measure Theory, Plenum Press, New York, NY, USA, 1992.

[25] T.-Y. Chen, "Optimistic and pessimistic decision making with dissonance reduction using interval-valued fuzzy sets," Information Sciences, vol. 181, no. 3, pp. 479-502, 2011. 


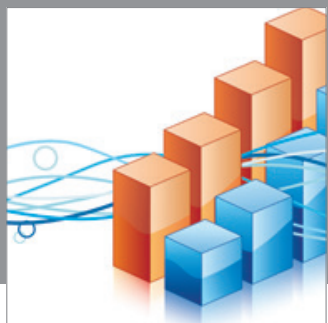

Advances in

Operations Research

mansans

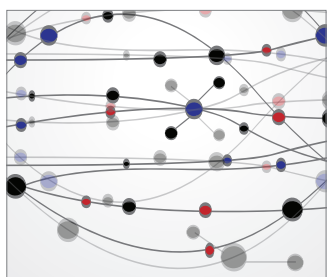

The Scientific World Journal
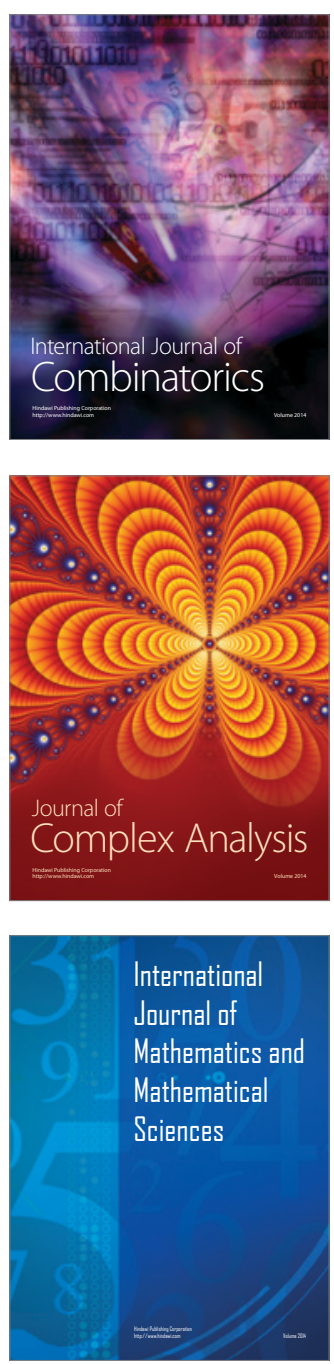
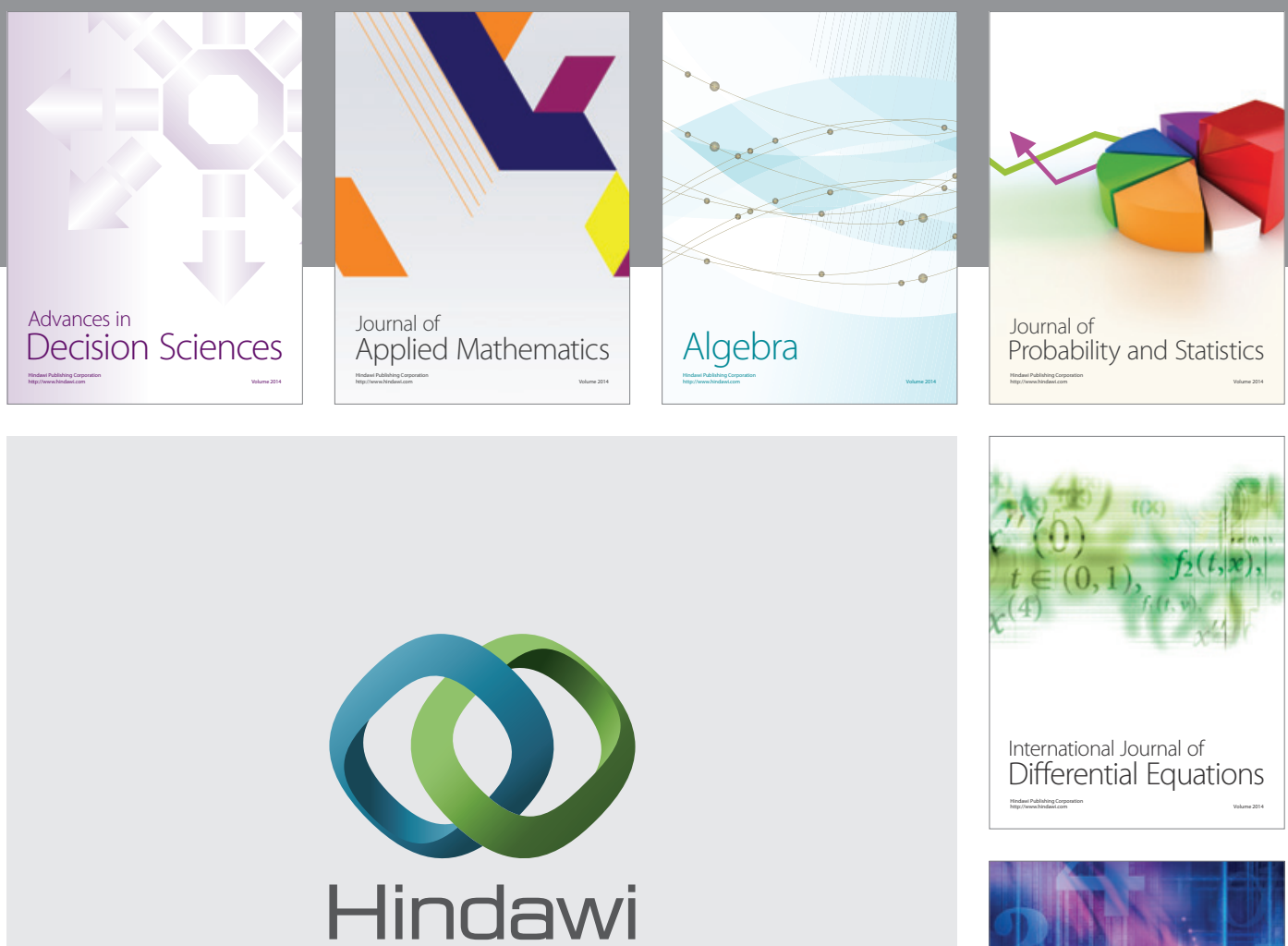

Submit your manuscripts at http://www.hindawi.com
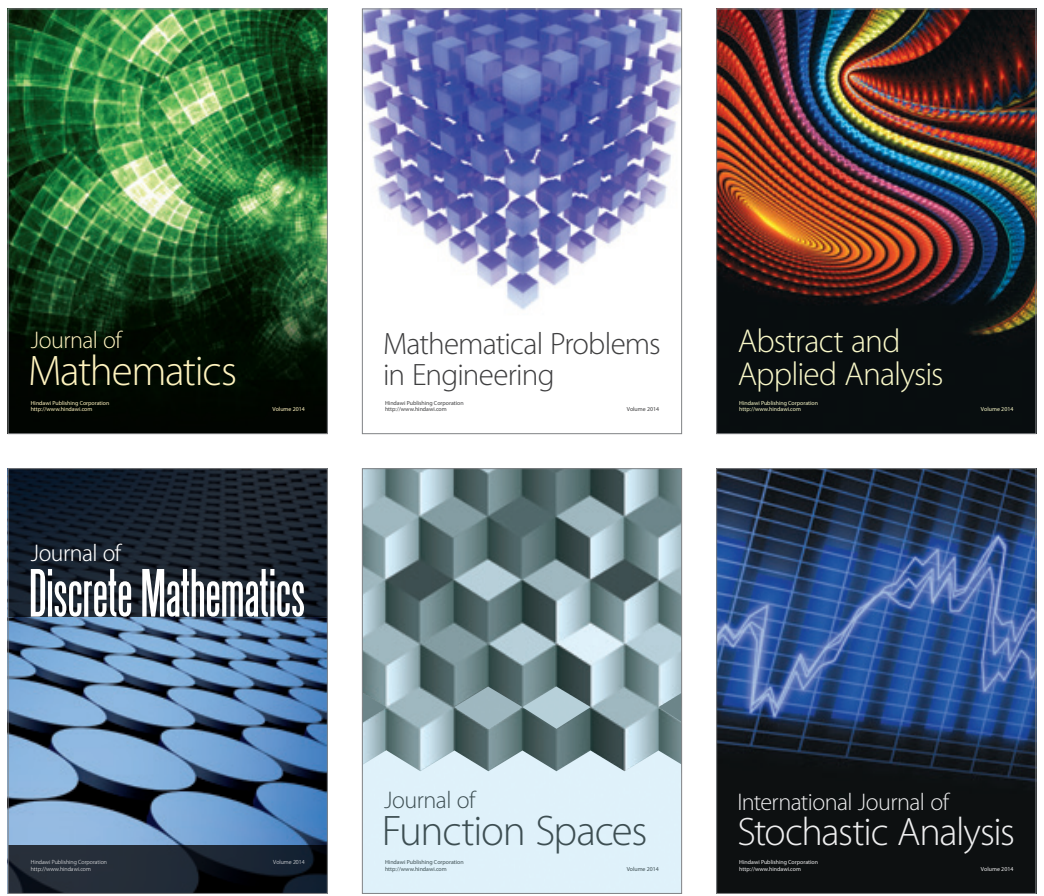

Journal of

Function Spaces

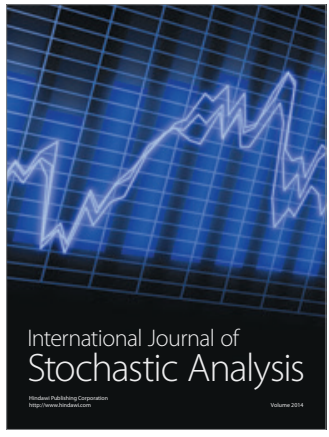

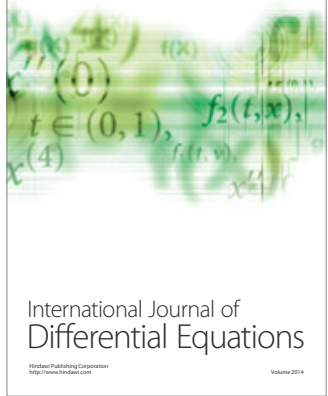
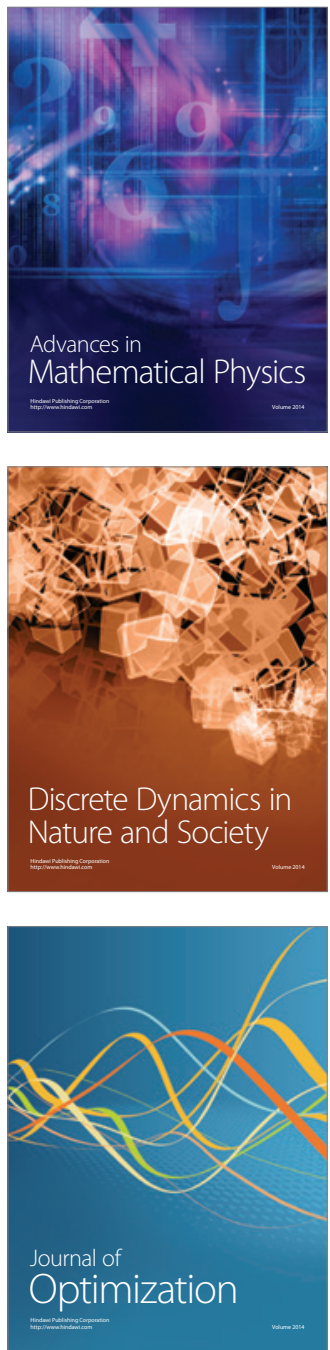